\title{
DEC1 is required for anti-apoptotic activity of gastric cancer cells under hypoxia by promoting Survivin expression
}

\author{
Yanfei Jia ${ }^{1}$ (i) $\cdot$ Rui $\mathrm{Hu}^{2} \cdot$ Ping $\mathrm{Li}^{3} \cdot$ Yan Zheng ${ }^{1} \cdot$ Yunshan Wang ${ }^{1,4} \cdot$ Xiaoli Ma ${ }^{1}$
}

Received: 27 July 2017 / Accepted: 15 November 2017 / Published online: 4 December 2017

(c) The International Gastric Cancer Association and The Japanese Gastric Cancer Association 2017

\begin{abstract}
Background Human differentiated embryonic chondrocyte-expressed gene 1 (DEC1), which has been reported to be overexpressed in several types of cancer, is associated with tumorigenesis through participation in several biological processes. However, the complex mechanisms underlying DEC1 during carcinogenesis are controversial, and its roles in the development and malignancy of gastric cancer (GC) remain unclear.

Methods We measured DEC1 expression in human GC cell lines. DEC1 levels in GC cells were downregulated by shRNA lentivirus infection. We also evaluated the effect of DEC1 downregulation on xenograft growth in vivo. The viability and apoptosis of the cells were assayed using the CCK8 assay and flow cytometry. The levels of DEC1, Survivin, and Bcl-2 were evaluated by Western blotting. Luciferase reporter was used to verify the downstream target of DEC1. The association of DEC1 and Survivin expression with prognosis was investigated by immunohistochemistry.

Results Downregulation of DEC1 inhibits GC cell proliferation in vitro and tumorigenicity in vivo. We observed that hypoxia-induced expression of DEC1 protects GC cells from apoptosis via transcriptional upregulation of Survivin. Furthermore, positive correlations between DEC1 with Survivin expression were observed in tissue sections from GC patients. Notably, GC patients with high expression levels of DEC1 and Survivin showed poor prognosis.

Conclusions DEC1 acts as an anti-apoptotic regulator in GC cells under hypoxia by promoting Survivin expression. Our study demonstrates the critical role of the DEC1 in oncogenesis and highlights a novel role for DEC1 in the regulation of cell apoptosis in GC.
\end{abstract}

Keywords DEC1 $\cdot$ Gastric cancer $\cdot$ Anti-apoptosis $\cdot$ Hypoxia $\cdot$ Survivin

Electronic supplementary material The online version of this article (https://doi.org/10.1007/s10120-017-0780-z) contains supplementary material, which is available to authorized users.

Yunshan Wang

sdjnwys@163.com

Xiaoli Ma

mx17125@126.com

1 Central Laboratory, Jinan Central Hospital

Affiliated to Shandong University, Jinan 250013,

People's Republic of China

2 Department of Reproduction, Jinan Central Hospital Affiliated to Shandong University, Jinan 250013, People's Republic of China

3 Department of Clinical Laboratory, Qilu Hospital of Shandong University, Jinan 250012, People's Republic of China

4 Shandong Province Key Lab of Tumor Target Molecule, Jinan Central Hospital Affiliated to Shandong University, Jinan 250013, People's Republic of China

\section{Introduction}

Gastric cancer (GC) accounts for a significant portion of cancer deaths, with a 5 -year survival rate of $15 \%$ being recorded [1]. The development of effective prevention and treatment strategies for GC is limited by an incomplete understanding of the signaling pathways involved in GC carcinogenesis [1].

Human differentiated embryonic chondrocyte-expressed gene 1 (DEC1) (also known as Bhlhe40/Bhlhb2/Stra13/ Sharp2) is a member of the basic helix-loop-helix (bHLH) transcription factor family and is critical in the development of chondrocyte cells and in the regulation of circadian rhythm [2-4]. Recent studies have indicated an important role of DEC1 in carcinogenesis [5]. The expression of DEC1 was determined to be positively associated with the incidence of oral squamous cell carcinoma (OSCC) and 1-year recurrence [6]. In breast cancer, DEC1 has been shown to 
be essential for the proliferation and promotion of tumor invasiveness [7, 8]. Conversely, nuclear DEC1 expression is reduced in hepatocellular carcinoma samples, showing decreased differentiation status [9]. Similarly, in pancreatic ductal adenocarcinoma, DEC1 has been reported to be a useful prognostic marker [10]. Whether DEC1 is upregulated or downregulated in lung cancer is controversial [11-14]. We were the first to report the high expression level of DEC1 in GC compared with normal gastric mucosa, and the DEC1 level was positively correlated with Ki67 expression [15, 16]. Therefore, DEC1 may act as a tumor promoter or tumor suppressor in different tumor tissue contexts. Because of this inconsistency, refined investigations are required to elucidate how DEC1 contributes to the development and progression of GC.

Accumulating evidence has demonstrated that hypoxia, irradiation, chemotherapy drugs, tumor necrosis factor (TNF)- $\alpha$, and transforming growth factor (TGF)- $\beta$ could induce DEC1 expression in cancer cells [5]. However, which of these factors is upstream or downstream of DEC1 in GC is not clear. Our previously study indicates that DEC1 is a hypoxia-induced gene and that its expression in GC may be a direct marker of tumor hypoxia [15, 17]. Given that adaptation to hypoxia is essential for solid tumor progression, it is intriguing to explore the regulatory function of DEC1 in hypoxic pathways [18]. Hypoxia produces a stress [20] ful environment for normal cells and can promote cell death. However, cancer cells are able to survive hypoxic environments, and hypoxia itself can activate adaptive cellular responses that contribute to tumor progression [19]. Thus, high expression of DEC1 in GC may be caused by hypoxic environments and may have an important effect on the survival of cancer cells under hypoxic conditions.

It has been shown that DEC1 is essential for anti-apoptotic effects in response to serum starvation and that these effects might be mediated through induction of Survivin expression in lung cancer [20]. Survivin is a member of the inhibitor of apoptosis proteins (IAP) family and has been implicated in inhibition of apoptosis in tumor cells and poor prognosis [21]. Furthermore, Zhang et al. have reported that Survivin expression could inhibit the apoptosis of hypoxic human pulmonary arterial smooth muscle cells [22]. Hypoxia has been observed to induce Survivin expression in lung and pancreatic cancer cells [23, 24]. The high expression of Survivin may reflect a low degree of cancer cell apoptosis under hypoxia. These findings led us to hypothesize that upregulation of DEC1 by hypoxia protects gastric cancer cells from apoptosis by promoting Survivin expression in GC.

In this study, we have identified a novel role for DEC1 in the anti-apoptotic survival mechanisms employed by cancer cells, which is an important step for tumor progression. Furthermore, we demonstrate that DEC1-induced anti-apoptotic effects were mediated via modulating Survivin expression in GC. This result suggests that targeting DEC1 could be a promising strategy for cancer therapy.

\section{Materials and methods}

\section{Cell culture}

NCI-N87, MKN28, MGC803, BGC823, SGC7901, AGS, and MKN45 cells (Chinese Academy of Sciences, Shanghai, China) were grown in $10 \%$ fetal bovine serum (FBS)-supplemented Dulbecco's modified Eagle's medium (DMEM; Gibco). Cells were maintained at $37^{\circ} \mathrm{C}$ under normoxic conditions in a $5 \% \mathrm{CO}_{2} / 95 \%$ ambient air incubator (Hera Cell 150; Heraeus, Germany) or hypoxic conditions in a modular incubator (Eppendorf, Germany) with settings at $0.5 \% \mathrm{O}_{2}$ $\left(5 \% \mathrm{CO}_{2}\right.$ and balance $\left.\mathrm{N}_{2}\right)$.

\section{Real-time PCR assays}

Total RNA was isolated using Trizol reagent (Invitrogen) according to the manufacturer's instructions. Total RNA was reverse transcribed using a Reverse Transcription Reaction Kit (Takara). Real-time polymerase chain reaction (PCR) was performed and analyzed on an Applied Biosystems 7300 Real-Time PCR System to determine the relative amounts of DEC1 and $\beta$-actin (internal control) mRNAs expressed. SYBR Green Supermix was used for all real-time PCR reactions. PCR primers used in this study are as follows: DEC1 Forward: ACTTACCTTGAAGCATGTGAAAGC A, Reverse: CATGTCTGGAAACCTGAGCAGAA; $\beta$-actin Forward: TGACGTGGACATCCGCAAAG, Reverse: CTG GAAGGTGGACAGCGAGG. Real-time PCR parameters were $95^{\circ} \mathrm{C}$ for $10 \mathrm{~s}$ as a pre-denaturing step followed by 40 PCR cycles of $95{ }^{\circ} \mathrm{C}$ for $5 \mathrm{~s}, 60{ }^{\circ} \mathrm{C}$ for $30 \mathrm{~s}$, and $72{ }^{\circ} \mathrm{C}$ for $10 \mathrm{~min}$. All samples were assayed in triplicate. The relative amount of mRNA was calculated using the comparative CT method after normalization to $\beta$-actin mRNA levels.

\section{Western blotting analysis}

Cell pellets were homogenized in extraction buffer [50 mmol/1 Tris- $\mathrm{HCl} \mathrm{pH} 6.8,0.1 \%$ sodium dodecyl sulfate (SDS), $150 \mu \mathrm{mol} / \mathrm{l} \mathrm{NaCl}, 100 \mathrm{mg} / \mathrm{l}$ phenylmethylsulfonyl fluoride, $1 \mathrm{mg} / \mathrm{l}$ aprotinin, $1 \% \mathrm{NP}-40$, and $0.5 \%$ sodium orthovanadate], incubated at $4{ }^{\circ} \mathrm{C}$ for $30 \mathrm{~min}$, and centrifuged for $20 \mathrm{~min}$ at $12,000 \mathrm{~g} / \mathrm{min}$. Total protein in the cell lysate was measured with a Bio-Rad colorimetric kit (Bio-Rad, Hercules, CA, USA). For Western blot analysis, total protein was separated on a $10 \%$ gel using SDS-PAGE and transferred onto nitrocellulose membranes $(0.45 \mu \mathrm{m}$; Millipore, Billerica, MA, USA), which were then incubated for $24 \mathrm{~h}$ at $4{ }^{\circ} \mathrm{C}$ 
with antibodies against DEC1 (1:300; Santa Cruz), HIF-1a (1:500; Abcam), Bcl-2 (1:500; Abcam), Survivin (1:500; Abcam), and $\beta$-actin (1:10,000; Abcam). Then, membranes were incubated with horseradish peroxidase-conjugated antimouse/rabbit IgG antibody (Santa Cruz) after a final wash. Signals were detected using an enhanced chemiluminescence kit (Amersham Pharmacia, Buckinghamshire, UK). $\beta$-Actin levels were used as an internal standard.

\section{Construction and infection of lentiviral vectors for modulating DEC1 expression}

Three different small interfering RNA (siRNA) sequences were tested for inhibitory activity against DEC 1 expression by transient transfection into BGC823 and MKN45 cells. The most effective sequence was cloned into the pGC-LV vector (Shanghai GeneChem, Shanghai, China). This short hairpin sequence specific for DEC1 (5'-CATTGCCCTGCA GAGTGGTTTACAACTT CCTGTCAGATTGTAAACC ACTCTGCAGGGCAATG- $3^{\prime}$ ) and a scrambled sequence (5'-TTCTCCGAACGTGTCACGCTTCCTGTC AGAACG TGACACGTTCGGAGAA-3') were annealed and inserted into the short hairpin RNA (shRNA) expression vector. Purified pGC-LV- DEC1, pHelper 1.0, and pHelper 2.0 plasmidtransfected $293 \mathrm{~T}$ cells were used to produce lentivirus. The supernatants of cultured cells were harvested after $48 \mathrm{~h}$ incubation with the lentivirus. Next, virus was purified with the plus-20 kit (Millipore, USA) and stored at $-80^{\circ} \mathrm{C}$. MKN45 and BGC 823 cells were infected with either DEC1-shRNA virus or scrambled NC-shRNA virus. All vectors expressed a green fluorescent protein (GFP) signal. Stable cells were generated using selection with $5 \mu \mathrm{g} / \mathrm{ml}$ puromycin (Sigma). The human DEC1 cDNA was subcloned into a pGV-puro lentiviral vector containing a puromycin resistance gene for establishment of stable cell lines (GeneChem, China). In this study, we pooled stable clones.

\section{Cell proliferation analysis}

Cell viability was determined using the CCK- 8 assay (Dojindo, Tokyo, Japan). Briefly, stably transfected cell lines were plated in $96-$ well plates (1500 cells/well). At daily intervals $(24,48,72$, and $96 \mathrm{~h}$ after plating) the cell proliferation assay was performed by the addition of $10 \mu \mathrm{l} \mathrm{CCK}-8$ solution to each well, followed by incubation at $37{ }^{\circ} \mathrm{C}$ for $2 \mathrm{~h}$. Absorbance was measured at a wavelength of $450 \mathrm{~nm}$ using a microplate reader (Bio-Rad, USA).

For cell proliferation analysis, $10^{4}$ cells were seeded into 6-well plates and serum starved for synchronization. After $48 \mathrm{~h}$, cells were stained with $2.5 \mathrm{EdU}$ dye (RiboBio, China) according to the supplier's recommendations.

\section{Colony formation assay}

Colony-forming capacity was assayed by seeding 1000 cells/ well in 12-well plates. Cells were cultured with DMEM plus $10 \%$ FBS and allowed to form colonies for 2-3 weeks in a $37{ }^{\circ} \mathrm{C}$ humidified atmosphere containing $5 \% \mathrm{CO}_{2}$. After removing the media and washing the cells with cold phosphate-buffered saline (PBS), colonies were fixed with methanol, washed in $\mathrm{H}_{2} \mathrm{O}_{2}$, and stained with $0.02 \%$ crystal violet.

\section{Apoptosis assay}

Apoptosis was measured using an annexin V/7-AAD (7-aminoactinomycine-D) kit (Becton-Dickinson). Briefly, the washed cell pellet was resuspended in $500 \mu \mathrm{l}$ binding buffer. Next, $5 \mu$ l Annexin-V-PE and $5 \mu 1$ 7-AAD were added. Flow cytometric analysis was performed immediately after staining. Data acquisition and analysis were performed with a Becton-Dickinson FACSCalibur flow cytometer using CellQuest software.

\section{In vivo assays for tumor growth}

MKN45 cells were stably infected with lenti-DEC1-shRNA or lenti-NC-shRNA vectors expressing green fluorescent protein $(\mathrm{GFP})$. Cells $\left(5 \times 10^{6}\right)$ were suspended in $50 \mu \mathrm{l}$ serum-free DMEM and implanted subcutaneously into the flanks of nude mice (six mice in each group; female BALB/c nu/nu, 4-6 weeks old). Nude mice were handled and cared for according to the NIH Animal Care and Use Committee guidelines in the Experiment Animal Center of the Medical School of Shandong University, China. All mice were monitored once every 3 days and killed after 5 weeks. Tumor onset was measured with calipers at the site of injection every 3 days by two trained laboratory staff members at different times on the same day, beginning 3 weeks after injection when an appreciable tumor volume began to form subcutaneously. Bioluminescence signal was measured using the IVIS Lumina system (Caliper Life Science, Hopkinton, MA, USA). Tumors were removed and embedded in paraffin. Tumor tissue sections were prepared, and immunohistochemical staining was analyzed using DEC1 (1:50; Genetex) and Survivin (1:500; Abcam) antibodies. Formalin-fixed tumors were analyzed for proliferation using EdU incorporation.

\section{Luciferase reporter assays}

Wild-type (WT) and mutant (MUT) Survivin promoter reporters were as described previously [20] and prepared with the pGL3-basic vector (Promega). MKN45 cells at 60\% confluence were seeded into 6-well plates and then transfected with the luciferase reporter gene construct (WT or 
MUT) and DEC1-overexpression vector. At $24 \mathrm{~h}$ after transfection, the cells were harvested and the Luc reporter assay was performed according to the manufacturer's instructions (Promega). All transfection experiments were conducted in triplicate and repeated thrice independently.

\section{Patients and samples}

Tissue microarrays (no. HStm-Ade180-Sur-02) containing 82 specimens of GC and para-carcinoma tissue were purchased from Xinchao Biotechnology (Shanghai, China). All the samples (each set of paired tumor and para-carcinoma tissues) were collected and categorized according to their clinical information from February 2008 to August 2008.

\section{Immunohistochemistry}

For immunohistochemical analysis, in brief, after deparaffinization, hydration, and antigen retrieval, the tissue sections were treated with endogenous peroxidase in $0.3 \% \mathrm{H}_{2} \mathrm{O}_{2}$ for $10 \mathrm{~min}$. Sections were blocked with $1.5 \%$ blocking serum in PBS and then incubated with primary rabbit polyclonal antibodies against DEC1 (1:50; Genetex) and Survivin (1:500; Abcam) at $4{ }^{\circ} \mathrm{C}$ overnight. The sections were then incubated with streptavidin-biotin-peroxidase complex/horseradish peroxidase (HRP) (Dako, Denmark) with 3,3'-diaminobenzidine (DAB) for 3 min and subsequently counterstained
A

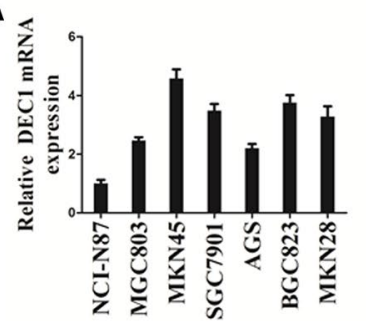

E

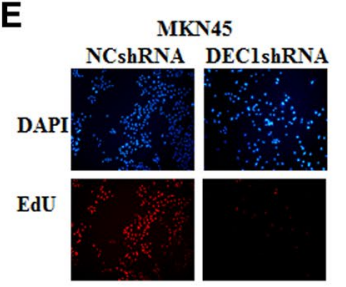

B

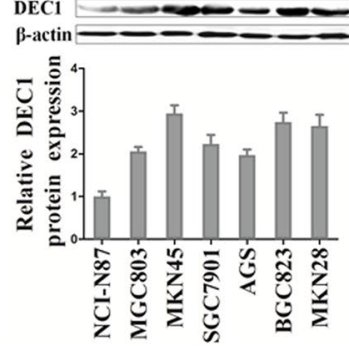

$\mathbf{F}$

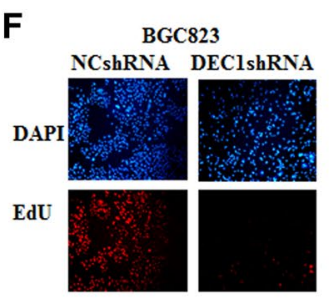

with hematoxylin. As a negative control, sections were incubated without primary antibody. The results were observed under a microscope. Assessment of DEC1 was performed as previously described by quantifying staining intensity and percentage of stained tumor cells. Expression was analyzed by two independent investigators who used a multi-headed microscope and were blinded to the clinical data.

\section{Results}

\section{DEC1 promotes GC cell proliferation in vitro and in vivo}

Because DEC1 expression has been reported to be upregulated in GC tissues [13], we investigated the possible effects of DEC1 on the growth of GC cells. We assayed the DEC1 expression level in seven GC cell lines by quantitative real-time (qRT)-PCR and Western blot analysis. Higher levels of DEC1 expression were observed in MKN45 and BGC823 cells (Fig. 1a, b). Next, MKN45 and BGC823 cells in which DEC1 was silenced or overexpressed were established. Quantitative cell viability analysis by the CCK-8 assay indicated that MKN45 and BGC823 cell viability steadily decreased following DEC1 knockdown (Fig. 1c, d; $P<0.01$ ), whereas overexpression had the opposite effect (Supplementary Fig. 1). In agreement with these results, the
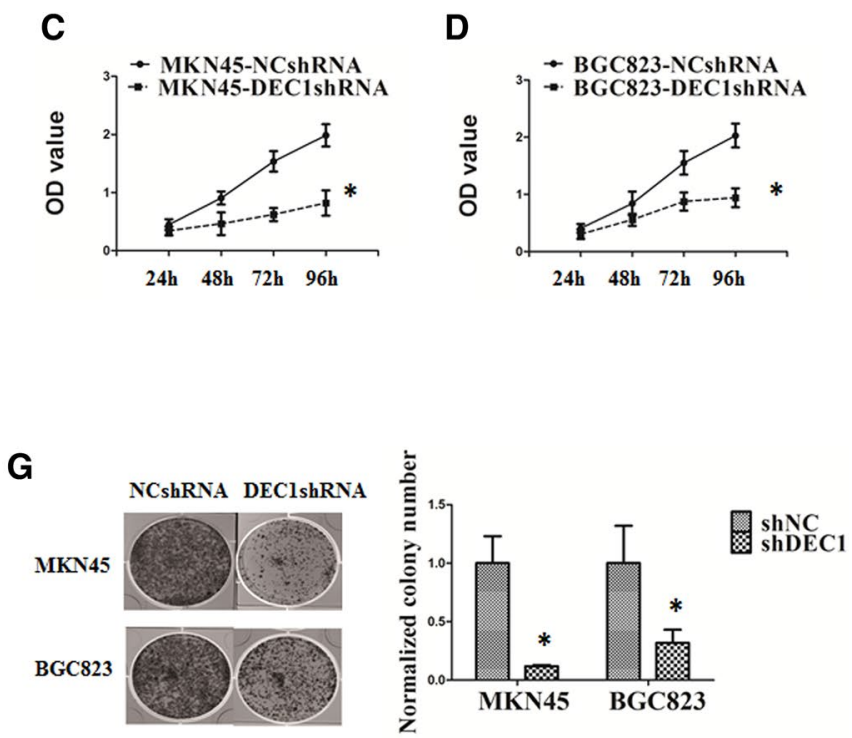

Fig. 1 Human differentiated embryonic chondrocyte-expressed gene 1 (DEC1) promotes gastric cancer (GC) cell proliferation in vitro. a Real-time polymerase chain reaction (RT-PCR) of DEC1 expression in GC cell lines. b Western blotting analysis of DEC1 protein level in GC cell lines. Quantification of immunoblots is shown in bottom panel. c, d Cell viability of MKN-45 and BGC823 cells was measured by CCK8 assay. e, f EdU incorporation assay shows that silenc- ing DEC1 in MKN-45 and BGC823 cells promoted cell proliferation. Red denotes positive spots and clusters; blue (DAPI) indicates nucleus. $\mathbf{g}$ Knockdown of DEC1 expression in MKN-45 and BGC823 cells decreased the numbers of tumor colonies in colony formation assays. Each bar in the histogram represents the mean \pm SD of three independent experiments. ${ }^{*} P<0.05$ 
fraction of proliferating cells having incorporated EdU was significantly lower in DEC1-shRNA-expressing cells compared with NC-shRNA-expressing cells (Fig. 1e, f). Colony formation assays also showed that DEC1 downregulation reduced the number and size of colonies formed by MKN45 and BGC 823 cells (Fig. 1g, h).

Having observed a functional role for DEC1 in regulating proliferation in GC cells, we next attempted to investigate the tumorigenic potential of DEC1 expression in vivo. MKN45 cells stably suppressing DEC1 and control cells were injected subcutaneously into nude mice. Tumors derived from DEC1-suppressing GC cell clones were smaller and weighed less than those derived from control cells (Fig. 2a-c). Western blot analysis confirmed DEC1 knockdown efficacy in DEC1-shRNA xenografts compared with the NC-shRNA group (Fig. 2d). Consistent with this finding, immunocytochemistry of sections obtained from tumors showed a reduction of DEC1 protein expression in DEC1-shRNA tumors compared with control sections (NC-shRNA) (Fig. 2e). DEC1-suppressing tumors showed a decrease in the number of proliferating cells, as revealed by EdU staining (Fig. 2f), consistent with in vitro data (Fig. 1). These results strongly emphasize the fundamental role of DEC1 in gastric tumor development.

\section{DEC1 maintain the survival of GC cells under hypoxia}

Survival under hypoxia is an important mechanism of solid tumor expansion. Given that hypoxia markedly induces the expression of DEC1, its expression is likely a response for survival of GC cells under hypoxia. To test this possibility, MKN45 and BGC823 cells were cultured for $72 \mathrm{~h}$ under hypoxic conditions. Cell viability was subsequently analyzed using the CCK- 8 assay, and apoptosis rate was detected by flow cytometry. Western blot analysis showed that hypoxia induced DEC1 expression in GC cells (Supplementary Fig. 2). MKN45 and BGC823 cell viability decreased under hypoxic conditions. Furthermore, a significant loss of viability was observed in MKN45-shDEC1 and BGC823-shDEC1 cells in hypoxia compared with either these cells in normoxia or control cells in hypoxia (Fig. 3a). However, overexpression of DEC1 blocked the hypoxia-induced loss of viability (Fig. 3a). In addition, DEC1 downregulation significantly increased the percentage of apoptotic cells in hypoxia (Fig. 3b).
A

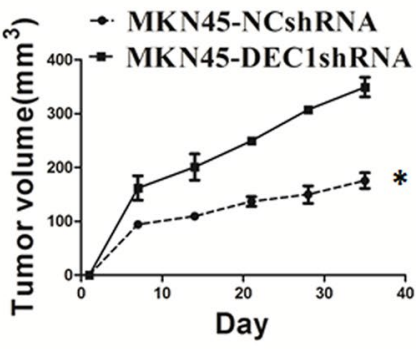

D

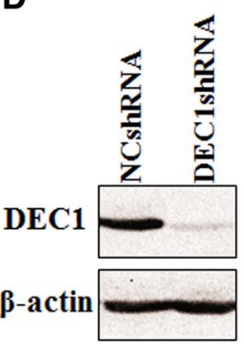

B

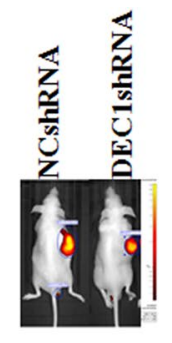

C

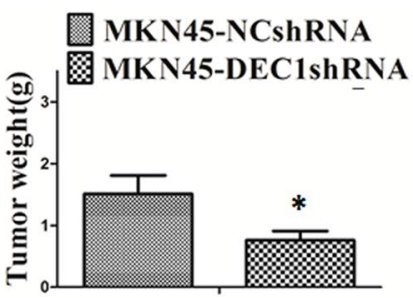

$\mathbf{E}$

NCshRNA

DEC1shRNA

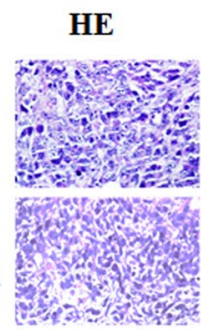

DEC1

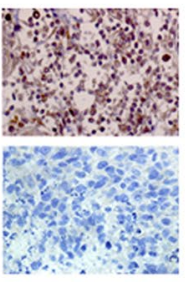

$\mathbf{F}$

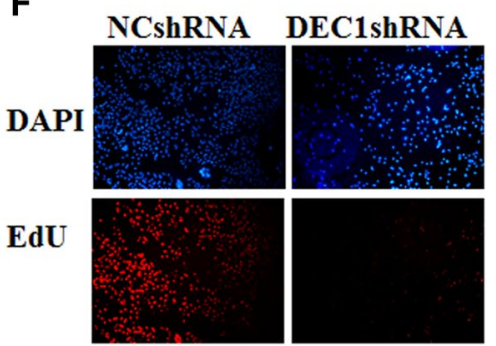

Fig. 2 Knockdown DEC1 in GC cells inhibited tumor growth and tumorigenesis in vivo. a Knockdown DEC1 in MKN45 cells dramatically inhibited tumor growth in nude mice. b Representative images of the xenograft tumors shown were obtained from the NCshRNA and DEC1 shRNA groups. c Mean weights of xenograft tumors. d Expression of DEC1 determined in xenograft tumors by Western blot analysis. $\beta$-actin was used as a loading control. e Representa- tive images for the expression of DEC1 xenograft tumor tissue sections determined using immunohistochemical (IHC) staining $(\times 400)$. f Sections of NCshRNA and DEC1shRNA tumors subjected to EdU analysis (representative images). Each experiment was independently performed at least three times. Data are presented as mean \pm SD. $* P<0.05$ 
Fig. 3 DEC1 maintains the survival of GC cells under hypoxia. a MKN-45 and BGC823 cells were grown under normoxic $\left(21 \% \mathrm{O}_{2}\right)$ or hypoxic $\left(0.5 \% \mathrm{O}_{2}\right)$ conditions for $72 \mathrm{~h}$. Proliferation was measured using CCK8. Values are mean \pm SD. b Apoptosis evaluated by Annexin V-PE versus 7-AAD flow cytometry analysis. Percentages of Annexin V+l PI- (early apoptotic cells) and Annexin V+/PI+ (late apoptotic cells) are shown. Images are representatives of one of three independent experiments with comparable results. Data are mean $\pm \mathrm{SD} . * P<0.05$
A
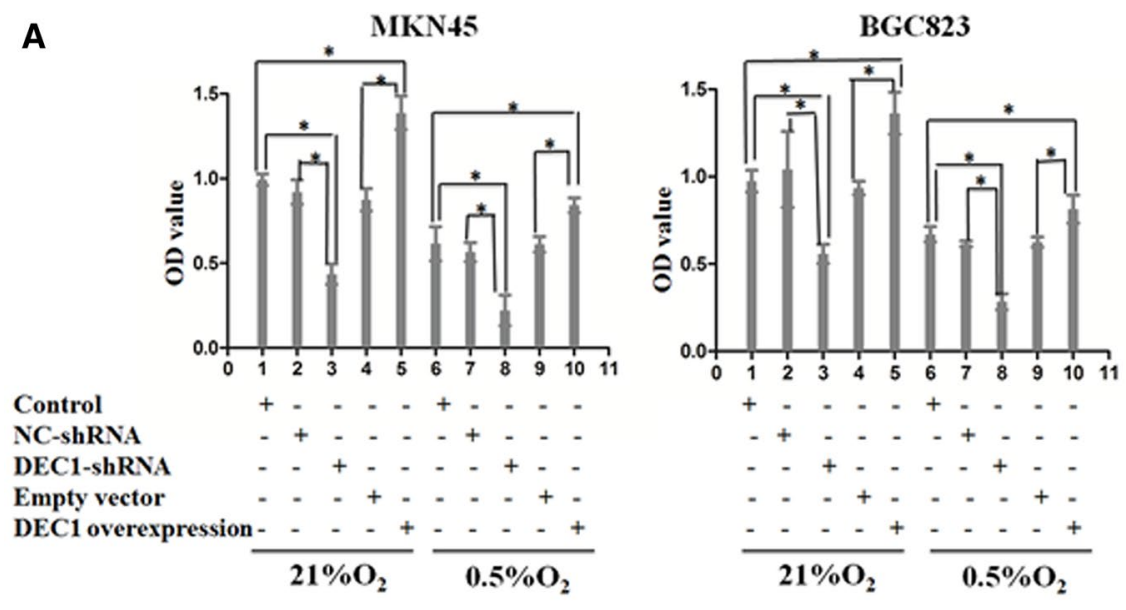

B
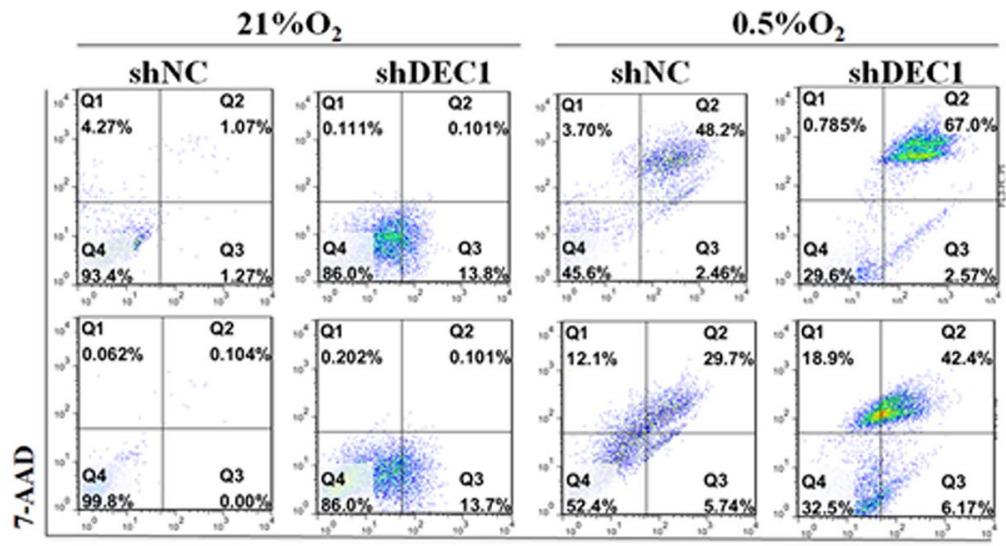

Annexin V

\section{Survivin contributes to anti-apoptotic mechanisms of DEC1 under hypoxia in GC}

Previous results showed that DEC1 inhibited serum starvation-induced apoptosis through upregulation of Survivin expression in lung cancer [15]. Therefore, we tested whether Survivin is involved in the anti-apoptotic effects of DEC1 under hypoxia. We confirmed that DEC1 knockdown dramatically reduced Survivin expression in normoxia (Fig. 4a). Conversely, overexpression of DEC1 induced Survivin expression (Fig. 4a). In the mouse model, tumors formed from DEC1-shNC, in which DEC1 is highly expressed, had more Survivin expression in comparison with the tumors from the DEC1-shRNA cells (Fig. 4b). Thus, it appears that Survivin expression is modulated by DEC1 expression. To verify whether Survivin is directly regulated by DEC1 activation in GC cells, a luciferase reporter assay was performed. Wild-type (WT) and mutant (MUT) luciferase reporter plasmids were constructed, differing in their binding sites. DEC1 overexpression substantially increased the activity of the reporter carrying the WT construct but not the MUT 3'-UTR of Survivin (Fig. 4c).
We further investigated whether Survivin inhibition could inhibit the oncogenic effects of DEC1 on GC cell proliferation under hypoxia. A significant loss of viability and increase in apoptosis was observed in DEC1-shRNA cells exposed to YM155, a Survivin inhibitor, compared with DEC1 knockdown alone in hypoxia (Fig. 5a, b). These data also demonstrate a decrease in Survivin and Bcl-2 protein expression in cells with DEC1 knockdown and exposed to YM155 treatment in hypoxic conditions, suggesting that Survivin contributes to the anti-apoptotic mechanisms of DEC1 under hypoxia in GC (Fig. 5c, d).

\section{DEC1 is highly expressed in human GC and associated with Survivin expression and decreased patient survival}

Immunohistochemical staining was performed to validate the correlation of DEC1 and Survivin expression in 82 human GC specimens and paired adjacent normal tissues. GC tissue exhibited greater immunoreactivity for DEC1 and Survivin antibodies compared with normal tissue (Fig. 6a, b). Multivariate analysis using the Cox proportional hazards model 


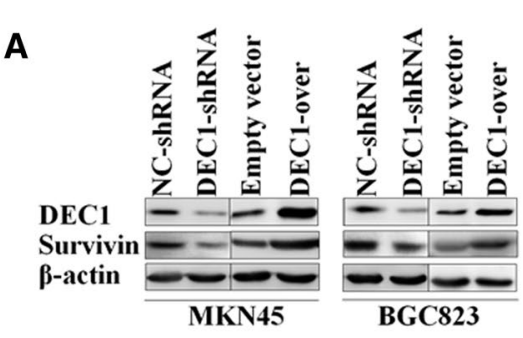

B

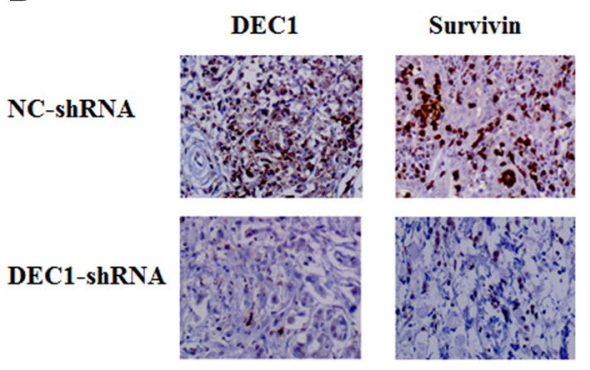

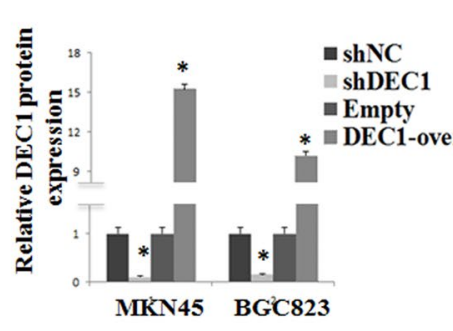

C

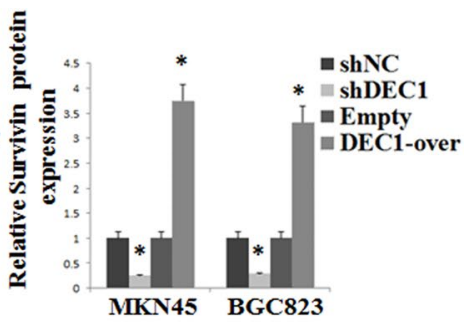


A

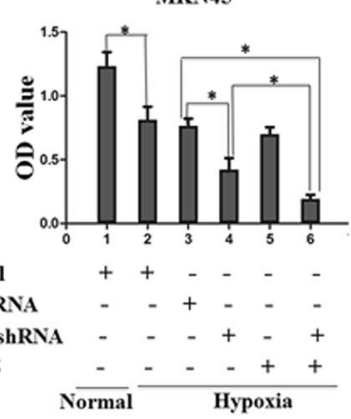

C

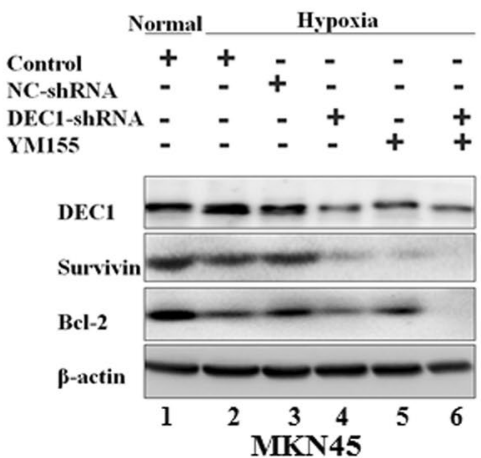

D

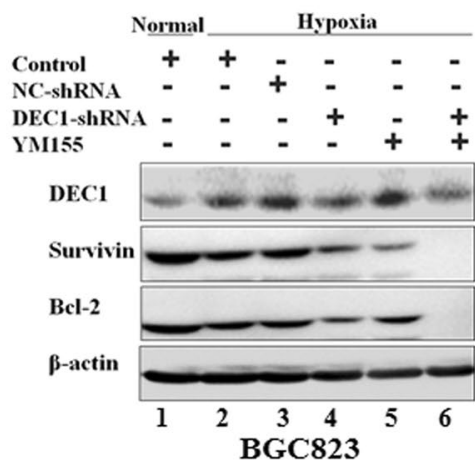

Fig. 5 Survivin contributes to anti-apoptotic mechanisms of DEC1 under hypoxia in GC. a, b DEC1 shRNA GC cells were treated with YM155, a Survivin inhibitor, under hypoxia for $72 \mathrm{~h}$. Cell viability

of anti-apoptotic proteins Bcl-2 and Survivin. In support of our data, DEC1 overexpression has been found to resist oxidative stress-mediated cell death in skeletal muscle and podocytes [31]. DEC1 also possesses anti-apoptotic effects in 8-MOP-treated HepG2 cells [32]. DEC1 is involved in the regulation of apoptosis by transforming growth factor treatment in mouse mammary carcinoma cells [25].

To date, the transcriptional regulation mechanisms of DEC1 in tumor progression remain controversial. DEC1 is generally considered to be transcriptionally repressive and to regulate DEC2 [33] and cyclin D1 [34] negatively. These trans-repression properties also indicate that DEC1 acts as a tumor suppressor in some tumor types. However,
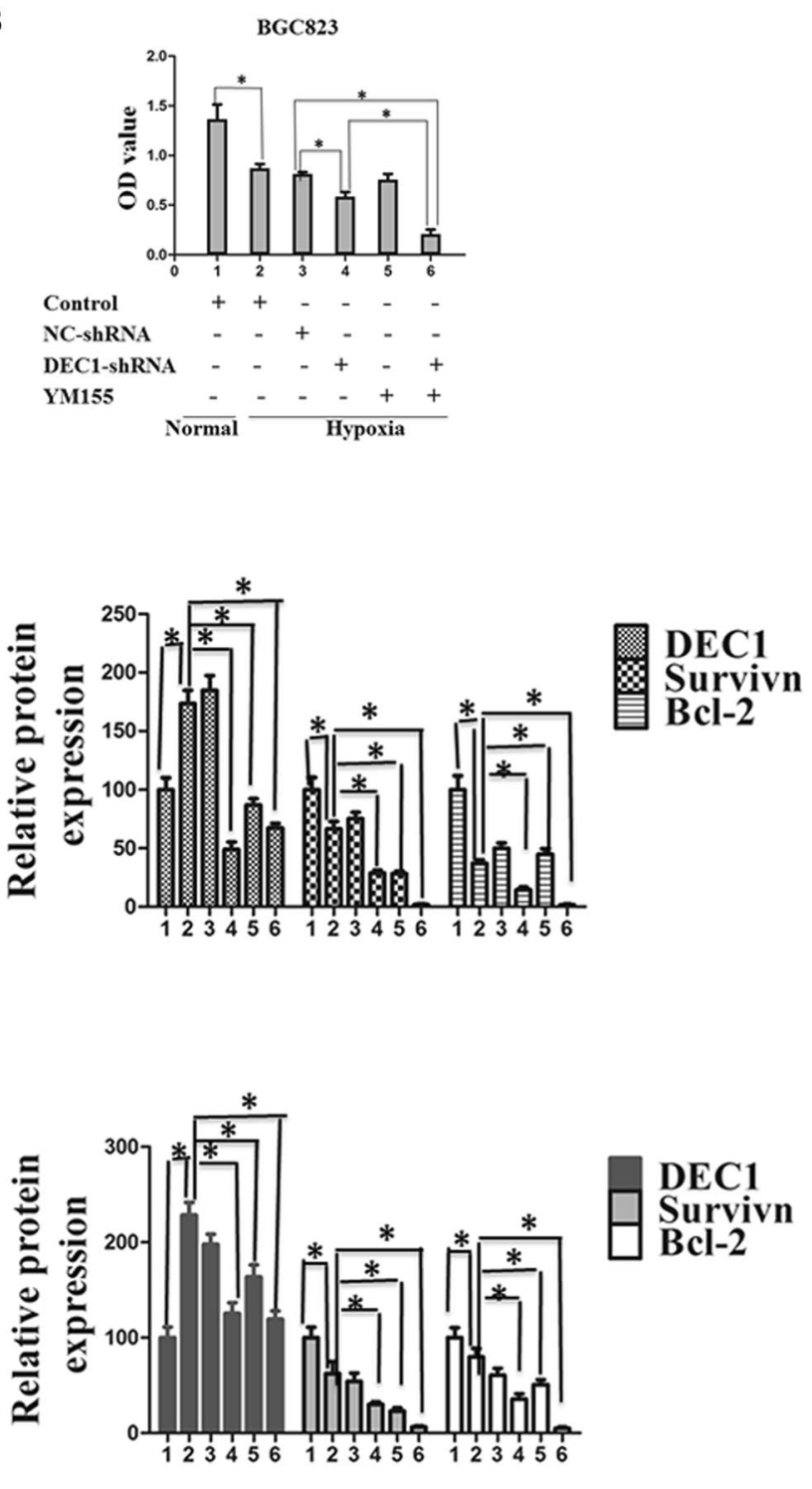

was assessed using the CCK8 assay. $\mathbf{c}, \mathbf{d}$ Western blotting analysis of DEC1, Survivin, and Bcl-2 protein level. $\beta$-Actin was used as a loading control. $* P<0.05$

DEC1 can act as a transcription activator to upregulate Survivin [20] and STAT3 [35] expression and is responsible for pro-tumor activities in certain contexts. To date, no studies have reported the transcriptional regulation role of DEC1 in GC. It is known that Survivin is of vital importance in the regulation of cell apoptosis in cancer cells [21]. Our study determined that Survivin expression is transcriptionally regulated by DEC1 both in vitro and in vivo. Furthermore, we demonstrated that inhibition of Survivin by a specific inhibitor increased apoptosis rate in cells with DEC1 knockdown in hypoxic conditions. The relevance of our study is highlighted by the results found in GC specimens. We showed that a high level of Survivin expression is correlated 


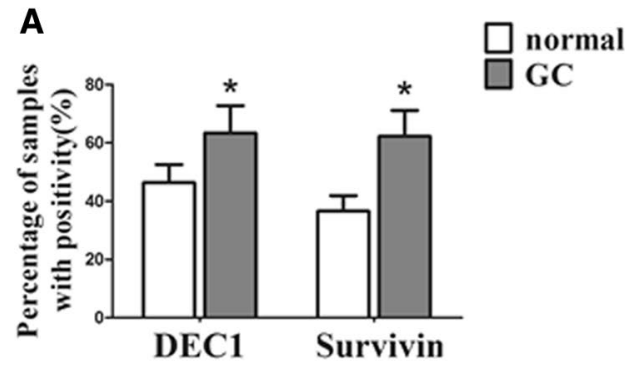

C

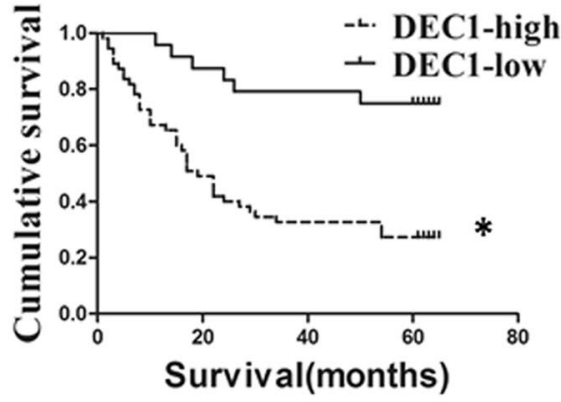

E

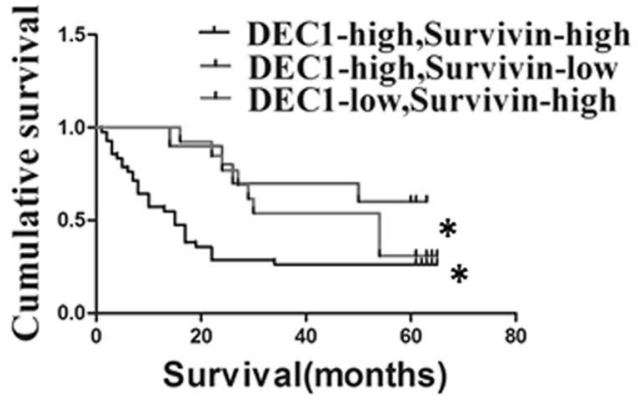

Fig. 6 DEC1 is highly expressed in human GC and is associated with Survivin expression and decreased patient survival. a DEC1 and Survivin expression levels were significantly upregulated in GC cancer tissues compared with normal tissues. b Representative IHC stain-

with DEC1 expression, as well as with poor patient survival. Thus, Survivin is an important target of DEC1 for GC patient survival. Therefore, our study also highlights a novel regulatory role in cell proliferation by DEC1 in GC.

Taken together, these results support a novel mechanism for DEC1 as an anti-apoptotic regulator in GC cells under hypoxia by promoting Survivin expression. Further exploration of the molecular link between DEC1 and the mechanism of cancer development may provide novel targets for cancer therapy.

Acknowledgments This work was financially supported by the National Natural Science Foundation of China (Nos. 31671468 and 81602593), the Chinese Postdoctoral Science Foundation (No.
B

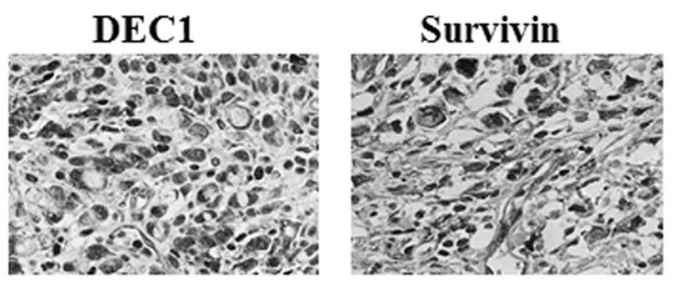

D

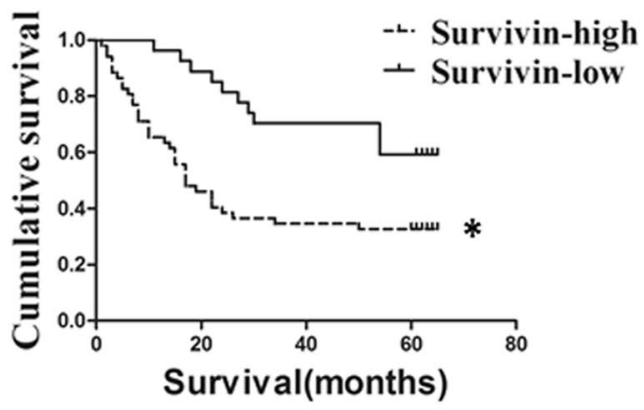

$\mathbf{F}$

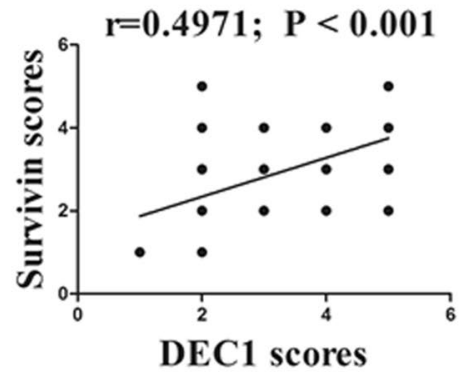

ing of DEC1 and Survivin in GC $(\times 400)$. c-e Overall survival curves of 82 GC patients segmented by DEC1, Survivin, and combination group. f Correlations between DEC1 and Survivin expression in GC. $* P<0.05$

2016M602152), the Shandong Provincial Natural Science Foundation of China (Nos. 2016ZRA01062, ZR2015HM018 and ZR2012HM061), and the Jinan Postdoctoral Innovation Project (172634).

\section{Compliance with ethical standards}

Conflict of interest The authors declare that they have no conflict of interest.

Informed consent statement All study participants, or their legal guardian, provided informed written consent before study enrollment. The Ethics Committee of Jinan Cental Hospital approved this study. 


\section{References}

1. Van Cutsem E, Sagaert X, Topal B, Haustermans K, Prenen H. Gastric cancer. Lancet. 2016;388:2654-64.

2. Shen M, Kawamoto T, Yan W, Nakamasu K, Tamagami M, Koyano Y, Noshiro M, Kato Y. Molecular characterization of the novel basic helix-loop-helix protein DEC1 expressed in differentiated human embryo chondrocytes. Biochem Biophys Res Commun. 1997;236:294-8.

3. Shen M, Yoshida E, Yan W, Kawamoto T, Suardita K, Koyano Y, Fujimoto K, Noshiro M, Kato Y. Basic helix-loop-helix protein DEC1 promotes chondrocyte differentiation at the early and terminal stages. J Biol Chem. 2002;277:50112-20.

4. Honma S, Kawamoto T, Takagi Y, Fujimoto K, Sato F, Noshiro M, Kato Y, Honma K. Dec1 and Dec2 are regulators of the mammalian molecular clock. Nature (Lond). 2002;419:841-4.

5. Sato F, Bhawal UK, Yoshimura T, Muragaki Y. DEC1 and DEC2 crosstalk between circadian rhythm and tumor progression. J Cancer. 2016;7:153-9.

6. You J, Lin L, Liu Q, Zhu T, Xia K, Su T. The correlation between the expression of differentiated embryo-chondrocyte expressed gene 1 and oral squamous cell carcinoma. Eur J Med Res. 2014;19:21.

7. Liu Y, Miao Y, Wang J, Lin X, Wang L, Xu HT, Wang EH. DEC1 is positively associated with the malignant phenotype of invasive breast cancers and negatively correlated with the expression of claudin-1. Int J Mol Med. 2013;31:855-60.

8. Chakrabarti J, Turley H, Campo L, Han C, Harris AL, Gatter KC, Fox SB. The transcription factor DEC1 (stra13, SHARP2) is associated with the hypoxic response and high tumour grade in human breast cancers. Br J Cancer. 2004;91:954-8.

9. Shi XH, Zheng Y, Sun Q, Cui J, Liu QH, Qu F, Wang YS. DEC1 nuclear expression: a marker of differentiation grade in hepatocellular carcinoma. World J Gastroenterol. 2011;17:2037-43.

10. Wang W, Reiser-Erkan C, Michalski CW, Raggi MC, Quan L, Yupei Z, Friess H, Erkan M, Kleeff J. Hypoxia inducible BHLHB2 is a novel and independent prognostic marker in pancreatic ductal adenocarcinoma. Biochem Biophys Res Commun. 2010;401:422-8.

11. Liu Y, Wang L, Lin XY, Wang J, Yu JH, Miao Y, Wang EH. The transcription factor DEC1 (BHLHE40/STRA13/SHARP-2) is negatively associated with TNM stage in non-small-cell lung cancer and inhibits the proliferation through cyclin D1 in A549 and BE1 cells. Tumour Biol. 2013;34:1641-50.

12. Falvella FS, Colombo F, Spinola M, Campiglio M, Pastorino U, Dragani TA. BHLHB3: a candidate tumor suppressor in lung cancer. Oncogene. 2008;27:3761-4.

13. Zhang L, Li QQ. Embryo-chondrocyte expressed gene 1, downregulating hypoxia-inducible factor 1 alpha, is another marker of lung tumor hypoxia. Acta Pharmacol Sin. 2007;28:549-58.

14. Giatromanolaki A, Koukourakis MI, Sivridis E, Turley H, Wykoff CC, Gatter KC, Harris AL. DEC1 (STRA13) protein expression relates to hypoxia- inducible factor 1-alpha and carbonic anhydrase- 9 overexpression in non-small cell lung cancer. J Pathol. 2003;200:222-8.

15. Jia YF, Xiao DJ, Ma XL, Song YY, Hu R, Kong Y, Zheng Y, Han SY, Hong RL, Wang YS. Differentiated embryonic chondrocyte-expressed gene 1 is associated with hypoxia-inducible factor 1alpha and Ki67 in human gastric cancer. Diagn Pathol. 2013;8:37.

16. Zheng Y, Jia Y, Wang Y, Wang M, Li B, Shi X, Ma X, Xiao $\mathrm{D}$, Sun Y. The hypoxia-regulated transcription factor DEC1 (Stra13, SHARP-2) and its expression in gastric cancer. OMICS. 2009;13:301-6.
17. Zheng Y, Shi X, Wang M, Jia Y, Li B, Zhang Y, Liu Q, Wang $Y$. The increased expression of DEC1 gene is related to HIF1alpha protein in gastric cancer cell lines. Mol Biol Rep. 2012;39:4229-36.

18. Qiu GZ, Jin MZ, Dai JX, Sun W, Feng JH, Jin WL. Reprogramming of the tumor in the hypoxic niche: the emerging concept and associated therapeutic strategies. Trends Pharmacol Sci. 2017;38(8):669-86.

19. Zeng W, Liu P, Pan W, Singh SR, Wei Y. Hypoxia and hypoxia inducible factors in tumor metabolism. Cancer Lett. 2015;356:263-7.

20. Li Y, Xie M, Yang J, Yang D, Deng R, Wan Y, Yan B. The expression of antiapoptotic protein survivin is transcriptionally upregulated by DEC1 primarily through multiple sp1 binding sites in the proximal promoter. Oncogene. 2006;25:3296-306.

21. Chen X, Duan N, Zhang C, Zhang W. Survivin and tumorigenesis: molecular mechanisms and therapeutic strategies. J Cancer. 2016;7:314-23.

22. Zhang S, Liu B, Fan Z, Wang D, Liu Y, Li J, Wang N, Liu Y, Zhang B. Targeted inhibition of survivin with YM155 promotes apoptosis of hypoxic human pulmonary arterial smooth muscle cells via the upregulation of voltage-dependent $\mathrm{K}(+)$ channels. Mol Med Rep. 2016;13:3415-22.

23. Harashima N, Takenaga K, Akimoto M, Harada M. HIF2alpha dictates the susceptibility of pancreatic cancer cells to TRAIL by regulating survivin expression. Oncotarget. 2017;8(26):42887-900.

24. Chen YQ, Zhao CL, Li W. Effect of hypoxia-inducible factor1alpha on transcription of survivin in non-small cell lung cancer. J Exp Clin Cancer Res. 2009;28:29.

25. Ehata S, Hanyu A, Hayashi M, Aburatani H, Kato Y, Fujime M, Saitoh M, Miyazawa K, Imamura T, Miyazono K. Transforming growth factor-beta promotes survival of mammary carcinoma cells through induction of antiapoptotic transcription factor DEC1. Cancer Res. 2007;67:9694-703.

26. Frigato E, Lunghi L, Ferretti ME, Biondi C, Bertolucci C. Evidence for circadian rhythms in human trophoblast cell line that persist in hypoxia. Biochem Biophys Res Commun. 2009;378:108-11.

27. Guillaumond F, Lacoche S, Dulong S, Grechez-Cassiau A, Filipski E, Li XM, Levi F, Berra E, Delaunay F, Teboul M. Altered Stra13 and Dec2 circadian gene expression in hypoxic cells. Biochem Biophys Res Commun. 2008;369:1184-9.

28. Ivanov SV, Salnikow K, Ivanova AV, Bai L, Lerman MI. Hypoxic repression of STAT1 and its downstream genes by a pVHL/HIF-1 target DEC1/STRA13. Oncogene. 2007;26:802-12.

29. Yamada K, Miyamoto K. Basic helix-loop-helix transcription factors, BHLHB2 and BHLHB3; their gene expressions are regulated by multiple extracellular stimuli. Front Biosci. 2005;10:3151-71.

30. Miyazaki K, Kawamoto T, Tanimoto K, Nishiyama M, Honda $\mathrm{H}$, Kato Y. Identification of functional hypoxia response elements in the promoter region of the DEC1 and DEC2 genes. J Biol Chem. 2002;277:47014-21.

31. Vercherat C, Chung TK, Yalcin S, Gulbagci N, Gopinadhan S, Ghaffari S, Taneja R. Stra13 regulates oxidative stress mediated skeletal muscle degeneration. Hum Mol Genet. 2009;18:4304-16.

32. Xiong J, Yang H, Luo W, Shan E, Liu J, Zhang F, Xi T, Yang J. The anti-metastatic effect of 8-MOP on hepatocellular carcinoma is potentiated by the down-regulation of bHLH transcription factor DEC1. Pharmacol Res. 2016;105:121-33.

33. Li Y, Xie M, Song X, Gragen S, Sachdeva K, Wan Y, Yan B. DEC1 negatively regulates the expression of DEC2 through 
binding to the E-box in the proximal promoter. J Biol Chem. 2003;278:16899-907.

34. Bhawal UK, Sato F, Arakawa Y, Fujimoto K, Kawamoto T, Tanimoto K, Ito Y, Sasahira T, Sakurai T, Kobayashi M, Kashima I, Kijima H, Kuniyasu H, Abiko Y, Kato Y, Sato S.
Basic helix-loop-helix transcription factor DEC1 negatively regulates cyclin D1. J Pathol. 2011;224:420-9.

35. Ivanova AV, Ivanov SV, Zhang X, Ivanov VN, Timofeeva OA, Lerman MI. STRA13 interacts with STAT3 and modulates transcription of STAT3-dependent targets. J Mol Biol. 2004;340:641-53. 